\title{
Complete tumor response in a patient with locally advanced lung large cell neuroendocrine carcinoma treated with Sintilimab plus platinum-based chemotherapy: a case report
}

Jinpeng Huang

Guangdong Provincial Hospital of Traditional Chinese Medicine

Feiye Wang

Guangdong Provincial Hospital of Traditional Chinese Medicine

Xiaohua Du

Guangdong Provincial Hospital of Traditional Chinese Medicine

Yongfen Li

Guangdong Provincial Hospital of Traditional Chinese Medicine

\section{Yuanyuan Zhuang}

Guangdong Provincial Hospital of Traditional Chinese Medicine

\section{Ziyan Gan}

Guangdong Provincial Hospital of Traditional Chinese Medicine

Shunqin Long

Guangdong Provincial Hospital of Traditional Chinese Medicine

Wanyin Wu

Guangdong Provincial Hospital of Traditional Chinese Medicine

Xiaobing Yang ( $\nabla$ yangxiaobing2002@126.com )

Guangdong Provincial Hospital of Traditional Chinese Medicine

\section{Case report}

Keywords: Lung large-cell carcinoma, PD-1 inhibitor, Chemotherapy, Complete response

Posted Date: May 17th, 2021

DOI: https://doi.org/10.21203/rs.3.rs-335963/v1

License: (c) (1) This work is licensed under a Creative Commons Attribution 4.0 International License.

Read Full License 


\section{Abstract \\ Background}

pulmonary large-cell neuroendocrine carcinoma (LCNEC) is a rare subset of lung cancer with a poor prognosis. As LCNEC is an uncommon malignancy, the optimal treatment for LCNEC has not been established.

\section{Case presentation}

: we report a case of a 66-year-old man diagnosed with unresectable, locally advanced LCNEC who presented a partial radiographic response to immunotherapy combined with chemotherapy. Salvage surgery was performed after 4 cycles of docetaxel and cisplatin (DP) regimen plus sintilimab, which is a highly selective, fully human antiprogrammed death-ligand 1 monoclonal antibody. Moreover, a pathologic analysis of the resected piece revealed the absence of residual viable tumor cells.

\section{Conclusion}

this case suggests that patients with locally advanced LCNEC may benefit from neoadjuvant chemoimmunotherapy, it is worthy for further study.

\section{Introduction}

Pulmonary large-cell neuroendocrine carcinoma (LCNEC) is an uncommon but destructive type of malignancy, demonstrated in a recent study to be more common among older adult males who have been smoking for decades ${ }^{1}$, comprising $<3 \%$ of all lung carcinoma ${ }^{2}$. Owing to its rarity, no extensive, prospective, and randomized clinical trials have been performed; choosing the optimal therapy regimen is challenging. In pathological performance, it covers the characteristics of small cell lung cancer (SCLC) and non-small cell lung cancer (NSCLC) ${ }^{3}$, making the clarification of its attribution controversial. In the2015 World Health Organization Classification of Lung Tumors, LCNEC was attributed to neuroendocrine tumor. The treatment strategy with reference to the National Comprehensive Cancer Network is based on the NSCLC project. There are no significant symptoms at the early stage; however, we detected it when it had developed to a locally advanced or advanced stage. LCNEC has a poor prognosis, with $<5 \%$ survival rates for stage IIIB-IV4. Surgery is still recommended, although not enough for the advanced types; therefore, neoadjuvant chemotherapy, radiotherapy, immune checkpoint inhibitors (ICls), etc., are needed.

Herein, we report a locally advanced LCNEC with complete tumor response. The patient accepted surgery after sintilimab plus platinum-based chemotherapy. This case suggests that patients with locally advanced LCNEC may benefit from neoadjuvant chemo-immunotherapy. 


\section{Case Presentation}

A 66-year-old man, who had a history of smoking, was admitted to our hospital with a 6-month history of cough with an Eastern Cooperative Oncology Group performance status of 1 point (ECOG 1) in June 2020. He had no significant medical history. A fluorodeoxyglucose positron emission tomography combined with computed tomography (FDG PET-CT) revealed a $6.5 \times 7.8 \times 7.0-\mathrm{cm}$ mass in the superior lobe of the right lung, with mediastinum invasion, right hilar lymph nodes, and mediastinal lymph node in station 4R metastases (Fig. 1). TNM classification (eighth edition) was cT4N2M0, consistent with stage $\mathrm{C} \mathbb{B}$. Then, he underwent a CT-guided percutaneous transthoracic needle aspiration biopsy, suggesting a poorly differentiated LCNEC. Immunohistochemistry also showed PD-L1 expression in 10\% of the cancer cells (Fig. 2), whereas no ALK or ROS-1 expression was observed. Next-generation sequencing (NGS) did not reveal any TKI-targetable mutation.

Therefore, the patient underwent a 4-cycle chemotherapy of docetaxel and cisplatin (DP) regimen combined with sintilimab (200 mg, every 3 weeks) from July 2020 to September 2020. After the treatment, chest CT scan images demonstrated a decrease in tumor size (Fig. 3). According to the guidelines of the Response Evaluation Criteria in Solid Tumors (version 1.1), the patient was considered to have a partial response.

In light of the impressive tumor response and considering the patient's young age and good ECOG performance status (ECOG 0 after the treatment), right upper lobectomy with right hilar lymph node and mediastinal lymph node dissection together with lysis of pleural adhesions were performed on October 16,2020 . A histological analysis of the resected lung and lymph nodes showed the absence of viable tumor cells (Fig. 4). Therefore, the pathological TNM classification (eighth edition) was ypT0 ypNO (R0). Sintilimab was continued after the surgery, and an additional 4-cycle chemotherapy of the DP regimen was planned.

Figure 1. Evaluation by FDG PET-CT at diagnosis. The mass in the superior lobe of the right lung was considered as the primary tumor.

Figure 2. Pathology at the time of diagnosis. Left: hematoxylin and eosin (HE) staining shows neoplastic cells with morphological characteristics of NSCLC. Right: immunohistochemistry reveals a diffuse expression of cytokeratin, leading to the diagnosis of lung LCNEC. Picture magnification: 20x; scale bar: $50 \mu \mathrm{m}$.

Figure 3. CT scan showing an decreased tumor size in the right lung.

Figure 4. At the time of surgery. Left: HE staining shows a phagocytic reaction in the necrotic area of former cancer tissue, with no residual viable cancer cells. Right: HE staining displays a collagen fiber hyperplasia in the necrotic area of former cancer tissue, with no residual viable cancer cells. Picture magnification: 20x; scale bar: $50 \mu \mathrm{m}$. Color online. 


\section{Discussion}

LCNEC is a pathological subtype of pulmonary neuroendocrine carcinoma, with the characteristics of high invasiveness and poor prognosis, and it is highly related to smoking ${ }^{5}$. LCNEC is a type of biologically aggressive cancer that behaves similarly to small cell carcinoma ${ }^{6}$. As most patients are diagnosed at the advanced stage, surgical treatment is limited, and the chances of recurrence are high. Therefore, systemic treatment is needed to improve the curative effect.

As LCNEC is an uncommon malignancy, the optimal treatment is unknown. The primary recommended treatment methods for LCNEC are the same as those recommended for NSCLC. Patients with early-stage LCNEC are primarily treated with surgery, and those at middle and advanced stages require multidisciplinary treatment. Most patients with early-stage LCNEC are treated with surgery ${ }^{7}$; however, surgery alone is insufficient to treat LCNEC. lyoda et al. retrospectively analyzed 335 cases of pathologic stage IA NSCLC and found that large-cell neuroendocrine histology is a significant adverse prognostic factor ${ }^{8}$. Therefore, surgical resection alone represents an insufficient treatment for LCNEC. Saji et al. retrospectively evaluated the efficacy of perioperative chemotherapy for patients with completely resected LCNEC and found that the patients who underwent surgery alone were 9.5 times more likely to die compared with patients who received surgery combined with chemotherapy. Therefore, perioperative chemotherapy is needed to improve survival in patients with $\mathrm{LCNEC}^{9}$. The most common chemotherapy used was the platinum-based combinations of VP-16 and CPT-11 ${ }^{9}$. Sun JM et al. evaluated whether an advanced LCNEC should be treated similarly to an SCLC or NSCLC. The treatment for advanced LCNEC, similar to SCLC, is more appropriate compared with that for NSCLC ${ }^{10} 11$.

LCNEC is a biologically heterogeneous group of tumors comprising distinct subsets based on genomic signatures: SCLC-like subset (TP53 + RB1 co-mutation/loss and other SCLC-type alterations), NSCLC-like subset (lack of co-altered TP53 + RB1 and a nearly universal occurrence of NSCLC-type mutations [STK11, KRAS, and KEAP1]), and carcinoid-like subset (MEN1 mutations and low mutation burden) ${ }^{12}$. The analysis of our patient's tumor by NGS revealed RB1 (c.1759G > T[p.E587*]) mutation, KEAP1 (c.1436A > T[p.D479V]) mutation, STK11 (c.616del[p.A206fs]) mutation, TP53 (c.230del[p.P77fs]) ERBB2 (c.3283G > A[p.A1095T]) mutation, SMARCA4 (c.2921C > T[p.P974L]) mutation, and SMAD4 (c.473T > A[p.V158E]) mutation. As the molecular characteristics of the patient accompanied with NSCLC-type (STK11 and KEAP1) and SCLC-type mutations (TP53 and RB1), we treated him with the DP regimen, which was effective for both diseases.

PD-1 inhibitors, such as pembrolizumab or nivolumab, are ICls that are proven to improve overall survival in advanced NSCLC ${ }^{13}$ 14; however, data about the efficacy of ICls in L-LCNEC are rare and limited mainly to some small sample clinical trials or a few case reports. Sherman et al. analyzed 37 patients with advanced LCNEC and found that patients treated with ICls had a longer median OS because advanced disease diagnosis was longer in patients treated with ICls than those who were not ${ }^{15}$. In this context of limited data on the efficacy of ICls in advanced LCNEC, little is known about their use in association with 
chemotherapy. The NADIM study indicated that neoadjuvant chemoimmunotherapy could change the perception of locally advanced NSCLC as a potentially lethal disease to one that is curable ${ }^{16}$. There is some data on the efficacy of neoadjuvant chemoimmunotherapy in patients with advanced LCNEC. Our patient with LCNEC received a complete tumor response after receiving sintilimab plus platinum-based chemotherapy. Sintilimab, a highly selective, fully human monoclonal antibody, blocks the interaction of PD-1 and its ligand, PD-L1. Compared with nivolumab or pembrolizumab, sintilimab has a different binding site and potentially greater affinity against PD- 1 as per the preclinical data ${ }^{17}$. The ORIENT- 11 study indicated the addition of sintilimab to chemotherapy with pemetrexed and platinum resulted in longer PFS than with chemotherapy alone in patients with advanced or metastatic nonsquamous NSCLC 18. Likewise, in our case, the postoperative pathology of the patient suggested a complete response, recommending that sintilimab and chemotherapy have a synergistic anticancer effect.

\section{Conclusion}

LCNEC is a rare subset of lung cancer with a poor prognosis. The efficacy of ICls in LCNEC has not yet been evaluated, but they might have certain advantages regarding survival. Immunotherapy combined with chemotherapy has been proven to prolong survival in patients with advanced NSCLC. In previous studies, neoadjuvant chemoimmunotherapy may turn some advanced NSCLC into curable diseases. However, further studies are still needed to confirm the role of neoadjuvant chemoimmunotherapy in advanced LCNEC.

\section{Declarations}

\section{Consent}

Written informed consent was obtained from the patient for publication of this Case report and any accompanying images. A copy of the written consent is available for review by the Editor-in-Chief of this journal.

\section{Author details}

a Medical Oncology Department, Guangdong Provincial Hospital of Chinese Medicine, The Second Clinical Medical College of Guangzhou University of Chinese Medicine; Guangzhou, Guangdong, People's Republic of China

b The Second Clinical Medical College of Guangzhou University of Chinese Medicine; Guangzhou, Guangdong, People's Republic of China

c Pathology Department, Guangdong Provincial Hospital of Chinese Medicine, The Second Clinical Medical College of Guangzhou University of Chinese Medicine; Guangzhou, Guangdong, People's 
Republic of China

d Thoracic Surgery Department, Guangdong Provincial Hospital of Chinese Medicine, The Second Clinical Medical College of Guangzhou University of Chinese Medicine; Guangzhou, Guangdong, People's Republic of China

\section{Funding}

This research did not receive any specific grant from funding agencies in the public, commercial, or notfor-profit sectors.

\section{Authors' contributions}

XBY and WYW: conception and design and final approval of the manuscript. JPH and FYW: wrote the first draft of the manuscript. XHD, YFL, ZYG, YYZ and SQL: collected the data. All authors read and approved the final manuscript.

\section{Competing interests}

The authors declare that they have no competing interests.

\section{Acknowledgements}

The language polishing of this article is helped by Innovent Biologics, Inc., People's Republic of China, and Eli Lilly and Company, United States.

\section{Abbreviations}

LCNEC: large-cell neuroendocrine carcinoma; SCLC: small cell lung cancer; NSCLC: non-small cell lung cancer; ICls: immune checkpoint inhibitors; PET-CT: positron emission tomography combined with computed tomography; NGS: Next-generation sequencing.

\section{References}

1. Gollard R, Jhatakia S, Elliott M, Kosty M: Large cell/neuroendocrine carcinoma. Lung Cancer 2010, 69:13-18.

2. Battafarano RJ, Fernandez FG, Ritter J, Meyers BF, Guthrie TJ, Cooper JD, Patterson GA: Large cell neuroendocrine carcinoma: an aggressive form of non-small cell lung cancer. J Thorac Cardiovasc Surg 2005, 130:166-172.

3. Travis WD, Brambilla E, Nicholson AG, Yatabe Y, Austin JHM, Beasley MB, Chirieac LR, Dacic S, Duhig E, Flieder DB, Geisinger K, Hirsch FR, Ishikawa Y, Kerr KM, Noguchi M, Pelosi G, Powell CA, Tsao MS, Wistuba I: The 2015 World Health Organization Classification of Lung Tumors: Impact of Genetic, Clinical and Radiologic Advances Since the 2004 Classification. J Thorac Oncol 2015, 10:1243-1260. 
4. Mauclet C, Duplaquet F, Pirard L, Rondelet B, Dupont M, Pop-Stanciu C, Vander Borght T, Remmelink M, D'Haene N, Lambin S, Wanet M, Remouchamps V, Ocak S: Complete tumor response of a locally advanced lung large-cell neuroendocrine carcinoma after palliative thoracic radiotherapy and immunotherapy with nivolumab. Lung Cancer 2019, 128:53-56.

5. Caplin ME, Baudin E, Ferolla P, Filosso P, Garcia-Yuste M, Lim E, Oberg K, Pelosi G, Perren A, Rossi RE, Travis WD: Pulmonary neuroendocrine (carcinoid) tumors: European Neuroendocrine Tumor Society expert consensus and recommendations for best practice for typical and atypical pulmonary carcinoids. Ann Oncol 2015, 26:1604-1620.

6. Asamura H, Kameya T, Matsuno Y, Noguchi M, Tada H, Ishikawa Y, Yokose T, Jiang SX, Inoue T, Nakagawa K, Tajima K, Nagai K: Neuroendocrine neoplasms of the lung:a prognostic spectrum. J Clin Oncol 2006, 24:70-76.

7. Gustafsson BI, Kidd M, Chan A, Malfertheiner MV, Modlin IM: Bronchopulmonary Neuroendocrine tumors. Cancer 2008, 113:5-21.

8. Iyoda A, Hiroshima K, Moriya Y, Sekine Y, Shibuya K, lizasa T, Nakatani Y, Fujisawa T: Prognostic impact of large cell neuroendocrine histology in patients with pathologic stage la pulmonary nonsmall cell carcinoma. J Thorac Cardiovasc Surg 2006, 132:312-315.

9. Saji H, Tsuboi M, Matsubayashi J, Miyajima K, Shimada Y, Imai K, Kato Y, Usuda J, Kajiwara N, Uchida O, Ohira T, Hirano T, Mukai K, Kato H, Ikeda N: Clinical response of large cell neuroendocrine carcinoma of the lung to perioperative adjuvant chemotherapy.Anticancer Drugs 2010, 21:89-93.

10. Sun JM, Ahn MJ, Ahn JS, Um SW, Kim H, Kim HK, Choi YS, Han J, Kim J, Kwon OJ, Shim YM, Park K: Chemotherapy for pulmonary large cell neuroendocrine carcinoma: Similar to that for small cell lung cancer or non-small cell lung cancer. Lung Cancer 2012, 77:365-370.

11. Derks JL, Hendriks LE, Buikhuisen WA, Groen HJ, Thunnissen E, van Suylen RJ, Houben R, Damhuis RA, Speel EJ, Dingemans AM: Clinical features of large cell neuroendocrine carcinoma: a populationbased overview. Eur Respir J 2016, 47:615 -624.

12. Rekhtman N, Pietanza MC, Hellmann MD, Naidoo J, Arora A, Won H, Halpenny DF, Wang H, Tian SK, Litvak AM, Paik PK, Drilon AE, Socci N, Poirier JT, Shen R, Berger MF, Moreira AL, Travis WD, Rudin CM, Ladanyi M: Next-Generation Sequencing of Pulmonary Large Cell Neuroendocrine Carcinoma Reveals Small Cell Carcinoma-like and Non-Small Cell Carcinoma-like Subsets. Clin Cancer Res 2016, 22: 3618-3629.

13. Borghaei H, Paz-Ares L, Horn L, Spigel DR, Steins M, Ready NE, Chow LQ, Vokes EE, Felip E, Holgado E, Barlesi F, Kohlhäufl M, Arrieta O, Burgio MA, Fayette J, Lena H, Poddubskaya E, Gerber DE, Gettinger SN, Rudin CM, Rizvi N, Crinò L, Blumenschein GR Jr, Antonia SJ, Dorange C, Harbison CT, Graf Finckenstein F, Brahmer JR: Nivolumab versus docetaxel in advanced non-squamous non-small cell lung cancer. N Engl J Med 2015, 373:1627-1639.

14. Herbst RS, Baas P, Kim DW, Felip E, Pérez-Gracia JL, Han JY, Molina J, Kim JH, Arvis CD, Ahn MJ, Majem M, Fidler MJ, de Castro G Jr, Garrido M, Lubiniecki GM, Shentu Y, Im E, Dolled-Filhart M, Garon 
EB. Pembrolizumab versus docetaxel for previously treated, PD-L1-positive, advanced non-small-cell lung cancer (KEYNOTE-010): a randomised controlled trial. Lancet 2016, 387:1540-1550,

15. Sherman S, Rotem O, Shochat T, Zer A, Dudnik E: Efficacy of immune check-point inhibitors (ICPi) in large cell neuroendocrine tumors of lung (LCNEC). Lung Cancer 2020, 143:40-46.

16. Provencio M, Nadal E, Insa A, García-Campelo MR, Casal-Rubio J, Dómine M, Majem M, RodríguezAbreu D, Martínez-Martí A, De Castro Carpeño J, Cobo M, López Vivanco G, Del Barco E, Bernabé Caro R, Viñolas N, Barneto Aranda I, Viteri S, Pereira E, Royuela A, Casarrubios M, Salas Antón C, Parra ER, Wistuba I, Calvo V, Laza-Briviesca R, Romero A, Massuti B, Cruz-Bermúdez A: Neoadjuvant chemotherapy and nivolumab in resectable non-small-cell lung cancer (NADIM): an open-label, multicentre, single-arm, phase 2 trial. Lancet Oncol 2020, 21:1413-1422.

17. Wang J, Fei K, Jing H, Wu Z, Wu W, Zhou S, Ni H, Chen B, Xiong Y, Liu Y, Peng B, Yu D, Jiang H, Liu J: Durable blockade of PD-1 signaling links preclinical efficacy of sintilimab to its clinical benefit. mAbs 2019, 11:1443-1451.

18. Yang Y, Wang Z, Fang J, Yu Q, Han B, Cang S, Chen G, Mei X, Yang Z, Ma R, Bi M, Ren X, Zhou J, Li B, Song Y, Feng J, Li J, He Z, Zhou R, Li W, Lu Y, Wang Y, Wang L, Yang N, Zhang Y, Yu Z, Zhao Y, Xie C, Cheng Y, Zhou H, Wang S, Zhu D, Zhang W, Zhang L: Efficacy and Safety of Sintilimab Plus Pemetrexed and Platinum as First-Line Treatment for Locally Advanced or Metastatic Nonsquamous NSCLC: a Randomized, Double-Blind, Phase 3 Study (Oncology pRogram by InnovENT anti-PD-1-11). J Thorac Oncol 2020, 15:1636-1646.

\section{Figures}
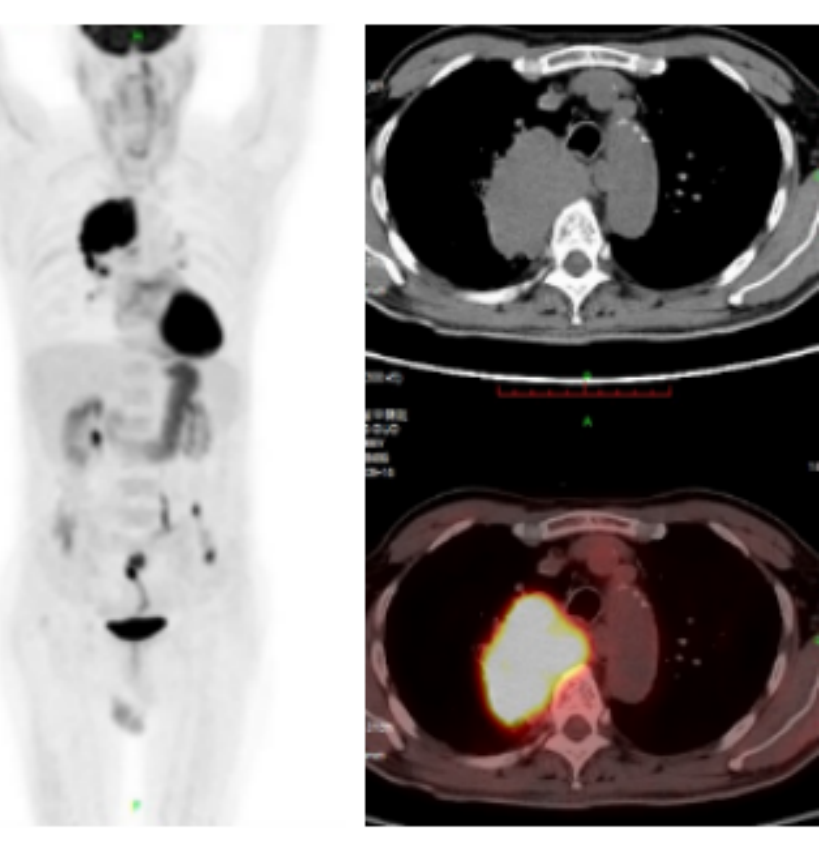

Figure 1 
Evaluation by FDG PET-CT at diagnosis. The mass in the superior lobe of the right lung was considered as the primary tumor.

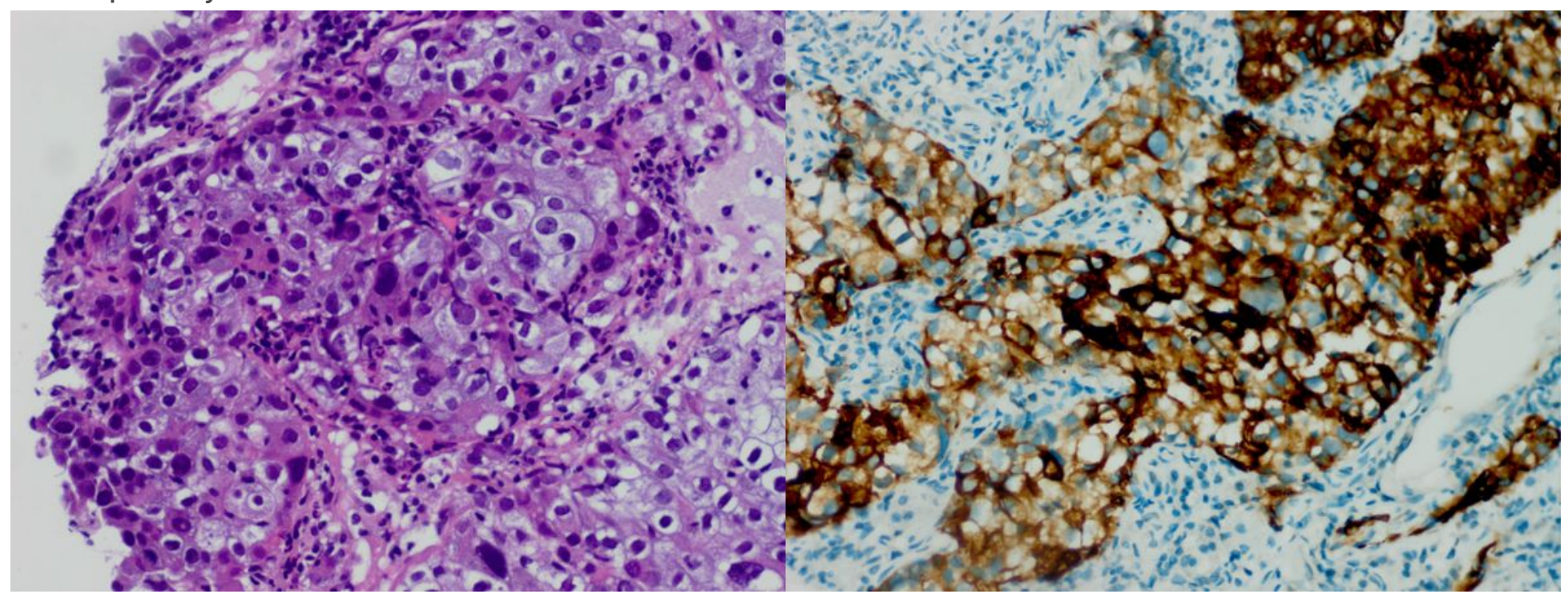

Figure 2

Pathology at the time of diagnosis. Left: hematoxylin and eosin (HE) staining shows neoplastic cells with morphological characteristics of NSCLC. Right: immunohistochemistry reveals a diffuse expression of cytokeratin, leading to the diagnosis of lung LCNEC. Picture magnification: 20x; scale bar: $50 \mu \mathrm{m}$.

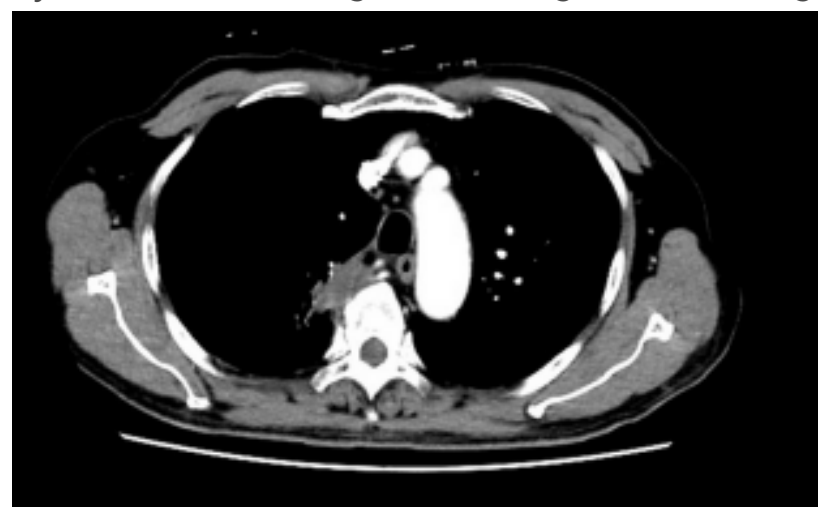

Figure 3

CT scan showing an decreased tumor size in the right lung. 


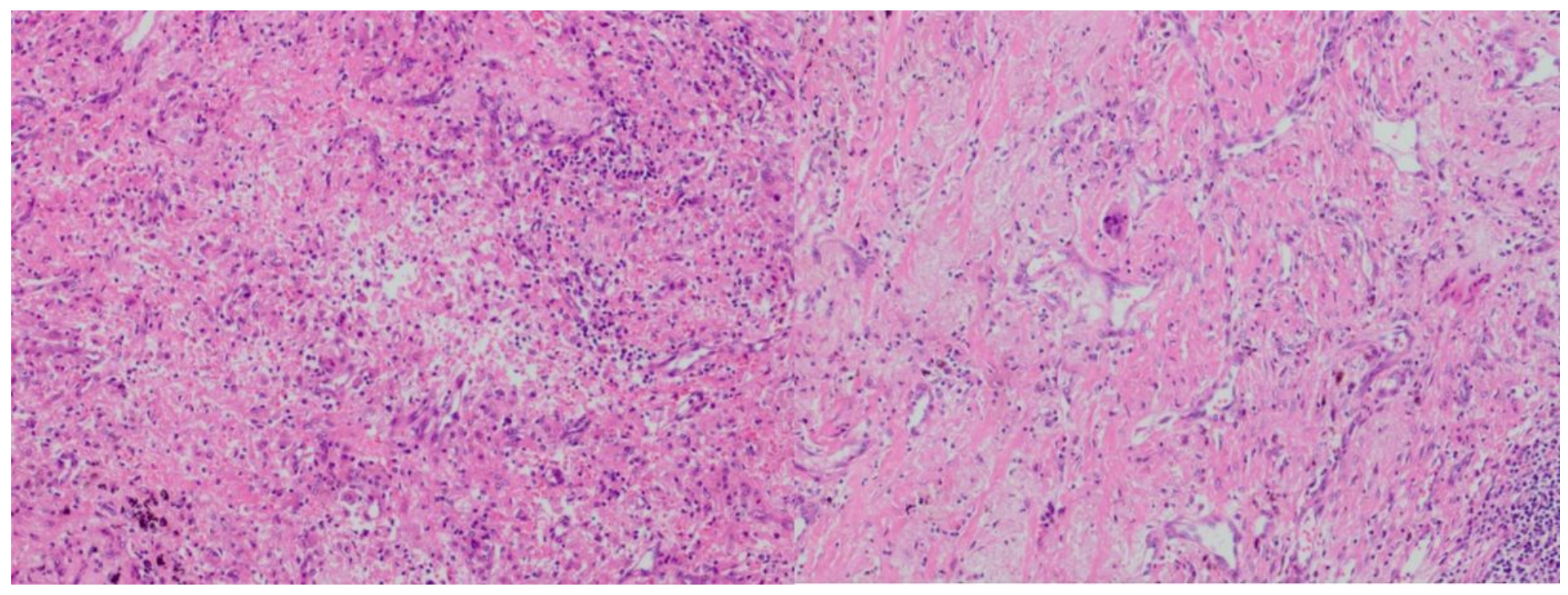

\section{Figure 4}

At the time of surgery. Left: HE staining shows a phagocytic reaction in the necrotic area of former cancer tissue, with no residual viable cancer cells. Right: HE staining displays a collagen fiber hyperplasia in the necrotic area of former cancer tissue, with no residual viable cancer cells. Picture magnification: 20x; scale bar: $50 \mu \mathrm{m}$. Color online. 